\section{Technical feasibility and efficacy of a standard needle magnetization system for ultrasound needle guidance in thyroid nodule-targeting punctures: a phantom study}

Haein Lee*, Yoon Joo Cho*, Eun Ju Ha, Jayoung Moon, You Na Kim, Minji Kim, Kyung-Min Lee, Sung Hyun An

Department of Radiology, Ajou University School of Medicine, Suwon, Korea

Purpose: The aim of this study was to assess the feasibility and efficacy of an ultrasound needle guidance system (NGS) based on standard needle magnetization in a phantom study of thyroid nodule (TN)-targeting punctures.

Methods: Six trainees and a staff radiologist performed TN-targeting punctures with or without the NGS in phantom models (group 1, experience $<50$ cases; group 2, experience $\geq 50$ cases and $<100$ cases; group 3, experience $\geq 100$ cases of TN-targeting punctures). The feasibility, technical success rate, number of punctures, and procedure time were recorded.

Results: The feasibility of NGS was $98.6 \%(138 / 140)$. In group 1, the technical success rate increased from $60.0 \% \pm 8.2 \%$ to $80.0 \% \pm 8.2 \%$ when the NGS was used $(P=0.046)$, with a reduction in the number of punctures from 2.2 to $1.2(P=0.005)$. In group 2 , the rate changed from $95.0 \% \pm 5.8 \%$ to $100.0 \% \pm 0.0 \%$ with the NGS ( $P=0.157)$, with a minimal decrease in the number of punctures from 1.1 to $1.0(P=0.157)$. The procedure time significantly decreased in both groups ( $P=0.041$ and $P=0.010$, respectively) when the NGS was used. In group 3, there were no significant differences in the technical success rate and the number of punctures according to whether the NGS was used $(P=0.317$ and $P=0.317$, respectively).

Conclusion: NGS using standard needle magnetization is technically feasible and has potential to improve the efficacy of TN-targeting punctures for less-experienced operators, especially beginners, according to the findings of this phantom study.

Keywords: Fine-needle aspiration; Needle guidance; Thyroid nodule; Ultrasound

Key points: The feasibility of electromagnetic needle guidance system (NGS) was $98.6 \%$ (138/140). NGS using the standard needle magnetization system could improve the technical success rate in thyroid nodule (TN)-targeting punctures in less-experienced operators. NGS using the standard needle magnetization system could decrease the number of punctures in TN-targeting punctures in less-experienced operators.

e-ultrasonography.org

Ultrasonography 41(3), July 2022

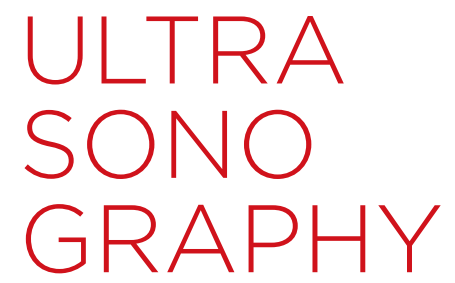

ORIGINAL ARTICLE

https://doi.org/10.14366/usg.21211 pISSN: 2288-5919 • elSSN: 2288-5943

Ultrasonography 2022;41:473-479

Received: October 13, 2021

Revised: December 9, 2021

Accepted: December 10, 2021

Correspondence to:

Eun Ju Ha, MD, Department of

Radiology, Ajou University School

of Medicine, 206 World cup-ro,

Yeongtong-gu, Suwon 16499, Korea

Tel. +82-31-219-4057

Fax. +82-31-219-5852

E-mail: radhej@naver.com

*These authors contributed equally to this work.

This is an Open Access article distributed under the terms of the Creative Commons Attribution NonCommercial License (http://creativecommons.org/ licenses/by-nc/4.0/) which permits unrestricted noncommercial use, distribution, and reproduction in any medium, provided the original work is properly cited.

Copyright (C) 2022 Korean Society of Ultrasound in Medicine (KSUM)

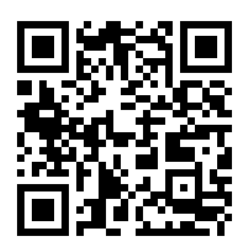

How to cite this article:

Lee H, Cho YJ, Ha EJ, Moon J, Kim YN, Kim $M$, et al. Technical feasibility and efficacy of a standard needle magnetization system for ultrasound needle guidance in thyroid nodule-targeting punctures: a phantom study. Ultrasonography. 2022 Jul;41(3):473-479. 


\section{Introduction}

Thyroid nodules (TNS) are a common clinical finding, with a detection rate of $17 \%-67 \%$ on ultrasonography (US) $[1,2]$. In patients with TNs, US-guided fine-needle aspiration (FNA) is the initial diagnostic tool, and the rate of malignancy ranges from $8 \%$ to $12 \%$ of nonpalpable nodules evaluated by FNA [1-3]. However, although US-guided FNA is a safe and accurate procedure for the diagnosis of TNs, there is a learning curve before the procedure can be performed optimally, particularly if the target structure is small or has a deep location [4-6]. Therefore, procedures performed by lessexperienced operators could generate a high rate of non-diagnostic results and increase patients' pain, discomfort, and procedurerelated complications [4-6].

To address this issue, US-guided needle-tracking and guidance systems have been developed to assist operators through continuous monitoring of the needle alignment and tip position during the procedure [7-16]. Conventional in-plane techniques allow operators to trace the entire length of the needle and the needle tip after insertion. However, these techniques have limitations for use if the insertion angle of the needle is misplaced and must be reinserted to target TNs [8]. Out-of-plane techniques allow the needle tip to be visualized when it crosses the US beam plane. However, these techniques generally require a higher level of experience, and the correct insertion angle also must be previously predicted [8]. Recently, a portable US device with an implemented needle guidance system (NGS) (SC1, FCU Co. Ltd., Daejeon, Korea) based on the standard needle magnetization system became commercially available. This system may have advantages for US-guided FNA of TNs because it can properly guide the direction and angle of the needle to the target nodules even before its insertion into the skin. Both in-plane and out-of-plane techniques can be performed safely with NGS assistance, if the system can easily magnetize a commercially available needle and accurately guide the exact insertion angle of the needle before performing skin punctures.

The present study assessed the technical feasibility and efficacy of US NGS using standard needle magnetization in a phantom study among residents being trained in $\mathrm{TN}$-targeting punctures compared to the conventional in-plane technique.

\section{Materials and Methods}

All punctures were performed by six radiology residents and a staff radiologist (group 1, H.L. and J.M. with $<50$ cases of experience with thyroid FNA; group 2, Y.N.K. and M.K. with $\geq 50$ cases and $<100$ cases of experience with thyroid FNA; and group 3, K.M.L., S.H.A., and E.J.H. with $\geq 100$ cases of experience with thyroid FNA) using phantom models. All operators were right-handed. Before the procedure, all metallic items that would potentially interfere with electromagnetic fields, such as watches, glasses, belts, cell phones, jewelry, detachable dental implants, and brassieres, were removed. Phantom models containing two targets with maximum diameters of $7 \mathrm{~mm}$ or $4 \mathrm{~mm}$ were used to mimic TN-targeting punctures (Fig. 1).

In the conventional technique without NGS, operators performed TN-targeting punctures with free hands in the phantom models. US examinations and US-guided TN-targeting punctures were

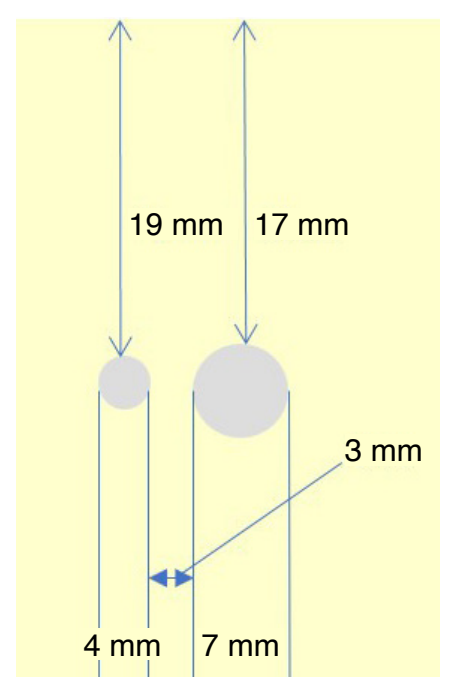

A

Fig. 1. Phantom model for needle puncture.

Schematic illustration (A), ultrasonography (B), and photograph (C) of the phantom show two targets $(7 \mathrm{~mm}$ and $4 \mathrm{~mm})$ mimicking thyroid nodules. 
performed using a 3-12 $\mathrm{MHz}$ linear probe and a real-time US system (RS85A, Samsung Medison Co. Ltd., Seoul, Korea). The TN-targeting punctures were performed using 23-gauge $25-\mathrm{mm}$ needles attached to a 10-mL syringe (Kovax-Needle, Korea Vaccine Co., Ltd., Seoul, Korea). In the NGS technique, a portable US device containing a 4-14 MHz linear transducer with NGS (SC 1, FCU Co., Ltd.) was used (Fig. 2). The transducer was calibrated to the background magnetic field of the intervention room according to the instructions of the implemented calibration application. Next, the same commercially available 23 -gauge $25-\mathrm{mm}$ needles attached to a 10-mL syringe were magnetized with a portable device. The technical feasibility assessment was focused on gaining an understanding of the standard needle magnetization system for ultrasound needle guidance and its applicability during the TN-targeting puncture. Therefore, the functionality of the standard needle magnetization system for ultrasound needle guidance was first assessed after a short (1-2 seconds) insertion of the needle into the magnetizer. Next, the quality of visualization and correlation of the needle tip and NGS on US during the procedure were assessed. After a short test of functionality, TNtargeting punctures were performed (Fig. 3).

Six radiology residents and a staff radiologist were asked to puncture each of the target nodules using a 23-gauge needle. A staff radiologist (E.J.H.) and a radiology assistant (Y.J.C.) judged whether the puncture procedure was successful and touched the center of the target nodule, and a research nurse (Y.J.K.) observed movement of the 23-gauge needle hub outside of the phantom and recorded the procedure time. Puncture success was achieved when the 23-gauge puncture needle touched the target nodule (Fig. 3). Puncture success for the target nodule on the first attempt was recorded as technical success. If the first attempt failed, it was repeated until successful and the total number of punctures was counted. Technical success rates of right-handed needling versus left-handed needling and 7-mm versus 4-mm target attempts were also recorded. The same process was repeated five times, and the entire process was also performed with the in-plane technique using a 5-12 MHz linear transducer and conventional US machine (RS85, Samsung Medison Co. Ltd.).

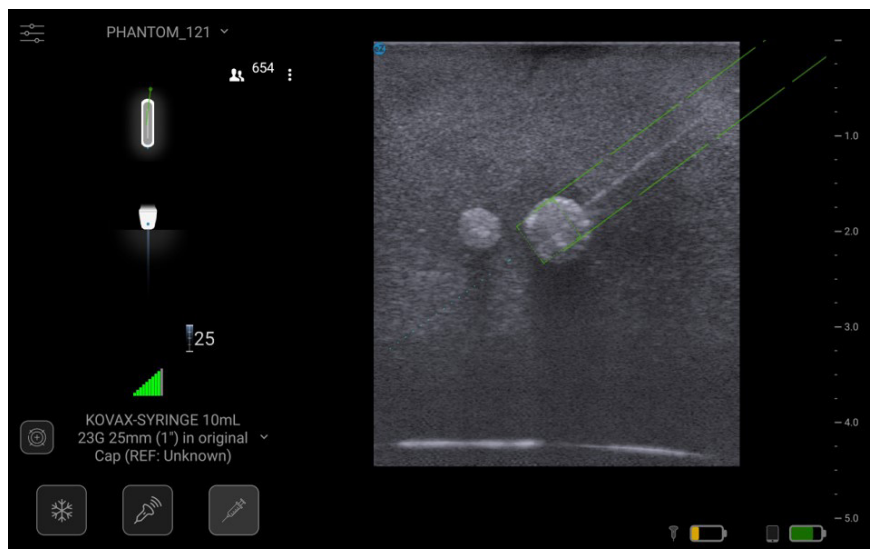

Fig. 3. Ultrasonography-guided procedure with a needle guidance system. The electromagnetic needle guidance system shows spatial information of the needle (dashed lines), as well as a target box superimposed on the currently acquired ultrasonographic images.

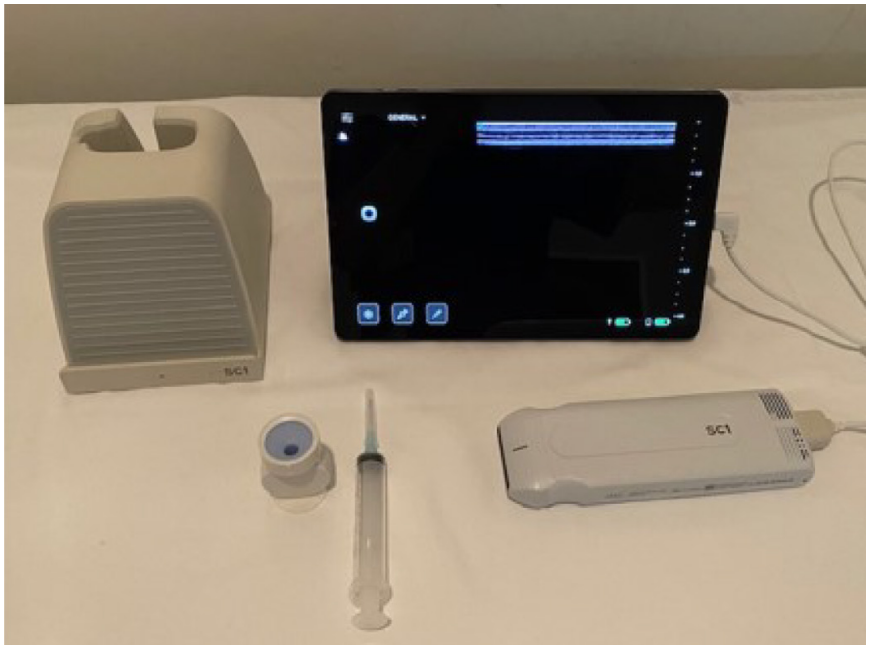

A

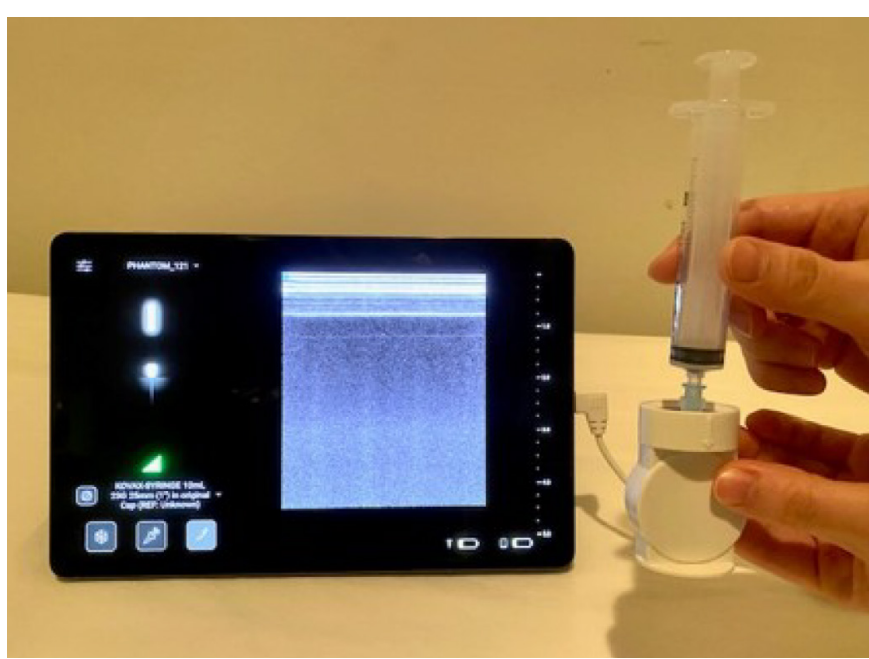

B

Fig. 2. Preparation of the needle guidance system.

A. A portable ultrasonographic apparatus with a standard needle magnetization system and a needle are used. B. After a brief insertion of the needle into the magnetizer (1-2 seconds), the performance indicator in the bottom left corner of the display shows full green bars, indicating good functionality of the system. 
Statistical analyses were conducted using SPSS version 25.0 (IBM Corp., Armonk, NY, USA) with the Wilcoxon signed-rank test to compare success rates between the conventional in-plane technique and the NGS-assisted technique using standard needle magnetization, left-handed needling and right-handed needling, and 7-mm and 4-mm target attempts. In all analyses, a P-value $<0.05$ was interpreted as indicating statistical significance.

\section{Results}

Technical Feasibility of a Standard Needle Magnetization System for Ultrasound Needle Guidance

The performance indicator showed good functionality after a short (1-2 seconds) insertion of the needle into the magnetizer in all cases (140/140). When the needle came close to the US transducer before its insertion into the skin, the NGS recognized the spatial orientation of the needle in relation to the US transducer in real time in all cases. During the targeting puncture, the technical feasibility of NGS using standard needle magnetization was $98.6 \%$ (138/140). Among the 140 attempts, the needle tip and NGS were well visualized and correlated during the procedure except for two attempts in which the direction of the needle and NGS did not fit well during the procedure, thus requiring an additional puncture for success.

\section{Efficacy of Ultrasound NGS in Targeting Punctures}

Table 1 compares the technical success rates between the conventional in-plane technique and the NGS-assisted technique using standard needle magnetization. In group 1, the technical success rate significantly increased from $60.0 \% \pm 8.2 \%$ to $80.0 \% \pm 8.2 \%$ when the NGS-assisted technique was used $(P=0.046)$, with a decrease in the mean number of punctures from 2.2 to $1.2(P=0.005)$. The rate was not significantly different for the NGS technique between left- and right-handed needling $(P=0.480)$ or between $7-\mathrm{mm}$ and 4 -mm target attempts $(P>0.99)$. In group 2 , the rate slightly increased from $95.0 \% \pm 5.8 \%$ to $100.0 \% \pm 0.0 \%$ with use of the NGS-assisted technique $(P=0.157)$, with a minimal decrease in the mean number of punctures from 1.1 to 1.0 $(P=0.157)$, although the differences were not statistically significant. Group 3 consistently showed excellent outcomes, without significant differences in the technical success rate and the number of punctures according to whether the NGS was used $(P=0.317$ and $P=0.317$, respectively). There were no differences between left- and right-handed needling or between 7-mm and 4-mm target attempts (all P>0.05).

Table 2 compares the procedure times between the techniques. In

Table 1. Comparison of the technical success rate and the number of punctures between the conventional in-plane technique and the technique assisted by the needle guidance system

\begin{tabular}{|c|c|c|c|c|c|c|c|c|c|}
\hline & \multicolumn{3}{|c|}{ Group 1} & \multicolumn{3}{|c|}{ Group 2} & \multicolumn{3}{|c|}{ Group 3} \\
\hline & Conventional & With NGS & P-value & Conventional & With NGS & P-value & Conventional & With NGS & P-value \\
\hline Technical success rate (\%) & $60.0(24 / 40)$ & $80.0(32 / 40)$ & 0.046 & $95.0(38 / 40)$ & $100(40 / 40)$ & 0.157 & $100(60 / 60)$ & $98.3(59 / 60)$ & 0.317 \\
\hline Right-handed needling & $60.0(12 / 20)$ & $85.0(17 / 20)$ & 0.059 & $90.0(18 / 20)$ & $100(20 / 20)$ & 0.157 & $100(30 / 30)$ & $100(30 / 30)$ & $>0.99$ \\
\hline $7 \mathrm{~mm}$ & $50.0(5 / 10)$ & $80.0(8 / 10)$ & 0.180 & $90.0(9 / 10)$ & $100(10 / 10)$ & 0.317 & $100(15 / 15)$ & $100(15 / 15)$ & $>0.99$ \\
\hline $4 \mathrm{~mm}$ & $70.0(7 / 10)$ & $90.0(9 / 10)$ & 0.157 & $90.0(9 / 10)$ & $100(10 / 10)$ & 0.317 & $100(15 / 15)$ & $100(15 / 15)$ & $>0.99$ \\
\hline Left-handed needling & $60.0(12 / 20)$ & $75.0(15 / 20)$ & 0.317 & $100(20 / 20)$ & $100(20 / 20)$ & $>0.99$ & $100(30 / 30)$ & $96.7(29 / 30)$ & 0.317 \\
\hline $7 \mathrm{~mm}$ & $60.0(6 / 10)$ & $80.0(8 / 10)$ & 0.157 & $100(10 / 10)$ & $100(10 / 10)$ & $>0.99$ & $100(15 / 15)$ & $93.3(14 / 15)$ & 0.317 \\
\hline $4 \mathrm{~mm}$ & $60.0(6 / 10)$ & $70.0(7 / 10)$ & 0.705 & $100(10 / 10)$ & $100(10 / 10)$ & $>0.99$ & $100(15 / 15)$ & $100(15 / 15)$ & $>0.99$ \\
\hline No. of punctures ${ }^{\mathrm{a})}$ & $2.2(87 / 40)$ & $1.2(48 / 40)$ & 0.005 & $1.1(42 / 40)$ & $1.0(40 / 40)$ & 0.157 & $1.0(60 / 60)$ & $1.0(61 / 60)$ & 0.317 \\
\hline Right-handed needling & $2.0(40 / 20)$ & $1.15(23 / 20)$ & 0.018 & $1.1(22 / 20)$ & $1.0(20 / 20)$ & 0.157 & $1.0(30 / 30)$ & $1.0(30 / 30)$ & $>0.99$ \\
\hline $7 \mathrm{~mm}$ & $2.2(22 / 10)$ & $1.2(12 / 10)$ & 0.068 & $1.1(11 / 10)$ & $1.0(10 / 10)$ & 0.317 & $1.0(15 / 15)$ & $1.0(15 / 15)$ & $>0.99$ \\
\hline $4 \mathrm{~mm}$ & $1.8(18 / 10)$ & $1.1(11 / 10)$ & 0.102 & $1.1(11 / 10)$ & $1.0(10 / 10)$ & 0.317 & $1.0(15 / 15)$ & $1.0(15 / 15)$ & $>0.99$ \\
\hline Left-handed needling & $2.4(47 / 20)$ & $1.3(25 / 20)$ & 0.103 & $1.0(20 / 20)$ & $1.0(20 / 20)$ & $>0.99$ & $1.0(30 / 30)$ & $1.0(31 / 30)$ & 0.317 \\
\hline $7 \mathrm{~mm}$ & $2.5(25 / 10)$ & $1.2(12 / 10)$ & 0.180 & $1.0(10 / 10)$ & $1.0(10 / 10)$ & $>0.99$ & $1.0(15 / 15)$ & $1.1(16 / 15)$ & 0.317 \\
\hline $4 \mathrm{~mm}$ & $2.2(22 / 10)$ & $1.3(13 / 10)$ & 0.380 & $1.0(10 / 10)$ & $1.0(10 / 10)$ & $>0.99$ & $1.0(15 / 15)$ & $1.0(15 / 15)$ & $>0.99$ \\
\hline
\end{tabular}

Group 1 indicates radiology residents with $<50$ cases of experience with thyroid fine-needle aspiration, while group 2 indicates radiology residents with $\geq 50$ cases and $<100$ cases of experience with thyroid fine-needle aspiration. Group 3 indicates experienced radiology residents and a staff radiologist with $\geq 100$ cases of experience with thyroid fine-needle aspiration.

NGS, needle guidance system.

${ }^{a}$ Numbers are mean values. 
Table 2. Comparison of the total procedure time between the conventional in-plane technique and the technique using the needle guidance system

\begin{tabular}{|c|c|c|c|c|c|c|c|c|c|}
\hline & \multicolumn{3}{|c|}{ Group 1} & \multicolumn{3}{|c|}{ Group 2} & \multicolumn{3}{|c|}{ Group 3} \\
\hline & Conventional & With NGS & P-value & Conventional & With NGS & P-value & Conventional & With NGS & P-value \\
\hline Procedure time (s) & $46.3 \pm 59.2$ & $24.4 \pm 23.1$ & 0.041 & $27.9 \pm 21.3$ & $17.6 \pm 7.9$ & 0.010 & $6.3 \pm 2.4$ & $9.0 \pm 3.6$ & $<0.001$ \\
\hline Right-handed needling & $53.4 \pm 59.7$ & $34.7 \pm 31.0$ & 0.027 & $25.6 \pm 19.9$ & $18.7 \pm 9.0$ & 0.109 & $5.6 \pm 2.0$ & $8.7 \pm 3.9$ & 0.001 \\
\hline $7 \mathrm{~mm}$ & $56.6 \pm 48.1$ & $31.1 \pm 38.3$ & 0.126 & $29.0 \pm 23.3$ & $20.0 \pm 7.0$ & 0.373 & $4.4 \pm 0.5$ & $6.0 \pm 1.7$ & 0.038 \\
\hline $4 \mathrm{~mm}$ & $50.1 \pm 72.0$ & $22.2 \pm 19.1$ & 0.123 & $22.1 \pm 16.3$ & $17.3 \pm 10.8$ & 0.138 & $6.8 \pm 2.3$ & $11.4 \pm 3.6$ & 0.005 \\
\hline Left-handed needling & $39.2 \pm 59.3$ & $22.2 \pm 14.2$ & 0.641 & $30.3 \pm 22.9$ & $16.5 \pm 6.8$ & 0.070 & $7.0 \pm 2.6$ & $9.2 \pm 3.4$ & 0.018 \\
\hline $7 \mathrm{~mm}$ & $45.0 \pm 67.5$ & $19.9 \pm 14.9$ & 0.359 & $34.8 \pm 21.5$ & $17.3 \pm 6.5$ & 0.074 & $6.6 \pm 1.8$ & $9.4 \pm 3.4$ & 0.131 \\
\hline $4 \mathrm{~mm}$ & $33.4 \pm 52.8$ & $24.5 \pm 13.8$ & 0.799 & $25.7 \pm 24.4$ & $15.6 \pm 7.3$ & 0.677 & $7.4 \pm 3.4$ & $9.0 \pm 3.7$ & 0.078 \\
\hline
\end{tabular}

Values are presented as mean \pm standard deviation. Group 1 indicates radiology residents with $<50$ cases of experience with thyroid fine-needle aspiration, while group 2 indicates radiology residents with $\geq 50$ cases of experience with thyroid fine-needle aspiration. Group 3 indicates experienced radiology residents and a staff radiologist with $\geq 100$ cases of experience with thyroid fine-needle aspiration.

NGS, needle guidance system.

groups 1 and 2, the time significantly decreased with the technique assisted by NGS ( $P=0.002)$. It decreased from $46.3 \pm 59.2$ seconds to $24.4 \pm 23.1$ seconds in group $1(P=0.041)$ and from $27.9 \pm 21.3$ seconds to $17.6 \pm 7.9$ seconds in group $2(\mathrm{P}=0.010)$ with the NGSassisted technique. However, in group 3, the time slightly increased from $6.3 \pm 2.4$ seconds to $9.0 \pm 3.6$ seconds when the NGS-assisted technique was used $(P<0.001)$.

\section{Discussion}

NGS using the standard needle magnetization system showed technical feasibility and efficacy for performing TN-targeting punctures in terms of improving the technical success rate while decreasing the number of punctures and procedure time for less-experienced operators. These observations indicate that the electromagnetic NGS can be useful for practical training in TNtargeting punctures, particularly for beginners.

The use of high-resolution US, combined with increased medical surveillance and access to health care services, has markedly increased the detection of TNs and number of FNAs $[1,2,17]$. Although FNA is an accurate and safe procedure for the diagnosis of TNs, sampling errors and procedure-associated complications have been reported [6]. Thyroid FNA is commonly performed at primary care centers by experienced radiologists, as well as many other clinicians, including endocrinologists, surgeons, family practice physicians, and other non-imaging specialists. Therefore, it is necessary to properly guide beginners and less-experienced operators in the performance of thyroid FNA in practice. Although it is necessary to track the needle from its insertion into the skin, its advancement through the soft tissues, and its entry into the target nodule in a long-axis approach, or at least the tip of the needle in a short-axis approach, accurate and consistent visualization of the needle alignment and tip is often difficult to achieve. In addition, insertion of the needle with an appropriate angle and direction into the skin is important to reduce targeting errors and minimize pain in the patient. Therefore, the conventional technique has a learning curve for operators. Electromagnetic needle tracking is a method that permits real-time needle spatial acquisition [8]. When the needle is located within a controlled magnetic field, voltage is induced in the sensor and used to calculate the position and orientation of the needle. In this study, we used a commercially available electromagnetic NGS in a portable US system for TNtargeting punctures to overcome the difficulty of determining the insertion angle of the needle and tracking the needle tip for targeting TNs. The system was easy to operate after magnetic polarization of the needle, and in almost all cases, it recognized the spatial orientation of the needle in relation to the US transducers in real time and guided the TN-targeting punctures before needle insertion.

The technical success rate significantly increased with the technique assisted by NGS, particularly in the beginners' group (experience $<50$ cases of TN-targeting punctures). This ultimately resulted in reductions in both the number of punctures and procedure-related time, thus indicating the potential of this system as an effective method to improve the puncture accuracy and to decrease procedure-related pain and complications in thyroid FNA. Although there were no significant differences in the experienced group ( $\geq 100$ cases of TN-targeting punctures), both hands can be easily used to handle the transducer and hold the needle, regardless of the target size and depth. Freesmeyer et al. similarly reported the technical effectiveness and safety of NGS based on the standard needle magnetization system in performing thyroid FNA [8]. They 
suggested that the appearance of the needle tip out-of-plane in combination with a color change in the NGS target box allows the operator to confirm the correct intranodular placement with confidence. As it is common for less-experienced operators to miss the anatomical targets, resulting in injuries to the vessels, trachea, and esophagus, we consider that this system would be effective for guiding less-experienced operators in performing thyroid FNA in the early learning period.

Several studies have reported the potential of electromagnetic NGS in many US-guided procedures, including biopsy, nerve block, spinal anesthesia, and central venous cannulation $[7,9-16,18]$. Kim et al. [11] reported that electromagnetic guidance significantly reduced the time required for needle placement during nerve block. In a randomized crossover study, Auyong et al. [10] demonstrated that real-time needle guidance technology significantly improved needle accuracy and performance time during cannulation of a simulated internal jugular vein in a phantom. In the present study, we developed a phantom for mimicking TN-targeting punctures. Studies were conducted by creating a situation similar to the actual depth and size of the nodules in the neck, and the results demonstrated its effectiveness. However, as performance in thyroid FNA can be affected by various factors, such as respiratory movements of the patient, swallowing, pulsating vessels, and neck muscles, as well as nodule-related factors, including location, size, and components, further studies using the NGS method in human subjects are required.

This study had several limitations. First, although the potential utility of NGS for less-experienced operators was demonstrated in this phantom study, it is uncertain whether NGS could improve the efficacy of FNA for less-experienced operators in real practice and would be helpful for training beginners in the free-hand FNA technique. However, this phantom study may suggest the potential utility of the NGS for beginners in accurate targeting and reducing the procedure time for FNA, which might shorten the learning curve and reduce the potential complication rate at an early stage of experience. Second, this study was conducted using one type of a commercially available 23 -gauge $25-\mathrm{mm}$ needle that we have used in practice for needle magnetization. Further studies are necessary to address these issues.

In conclusion, NGS using standard needle magnetization is technically feasible and has the potential to improve the efficacy of TN-targeting punctures for less-experienced operators, especially beginners, based on the findings of this phantom study.

ORCID: Haein Lee: https://orcid.org/0000-0002-2020-6888; Yoon Joo Cho: https:// orcid.org/0000-0002-1608-8938; Eun Ju Ha: https://orcid.org/0000-0002-12342919; Jayoung Moon: https://orcid.org/0000-0003-4722-3423; You Na Kim: https:// orcid.org/0000-0003-2771-2182; Minji Kim: https://orcid.org/0000-0002-83917476; Kyung-Min Lee: https://orcid.org/0000-0002-0361-0942; Sung Hyun An: https://orcid.org/0000-0003-2533-7227

\section{Author Contributions}

Conceptualization: Ha EJ. Data acquisition: Lee H, Cho YJ, Ha EJ, Moon J, Kim YN, Kim M, Kim KM, An SH. Data analysis or interpretation: Lee H, Cho YJ, Ha EJ. Drafting of the manuscript: Lee H, Cho YJ, Ha EJ, Moon J, Kim YN, Kim M. Critical revision of the manuscript: Ha EJ. Approval of the final version of the manuscript: all authors.

\section{Conflict of Interest}

No potential conflict of interest relevant to this article was reported.

\section{Acknowledgments}

This research was supported by a grant of the Korea Health Technology R\&D Project through the Korea Health Industry Development Institute (KHIDI), funded by the Ministry of Health \& Welfare, Republic of Korea (grant number: HI20C2140).

\section{References}

1. Shin JH, Baek JH, Chung J, Ha EJ, Kim JH, Lee YH, et al. Ultrasonography diagnosis and imaging-based management of thyroid nodules: revised Korean Society of Thyroid Radiology Consensus Statement and Recommendations. Korean J Radiol 2016;17:370-395.

2. Haugen BR, Alexander EK, Bible KC, Doherty GM, Mandel SJ, Nikiforov YE, et al. 2015 American Thyroid Association management guidelines for adult patients with thyroid nodules and differentiated thyroid cancer: the American Thyroid Association Guidelines Task Force on Thyroid Nodules and Differentiated Thyroid Cancer. Thyroid 2016;26:1-133.

3. Ha EJ, Na DG, Baek JH. Korean Thyroid Imaging Reporting and Data System: current status, challenges, and future perspectives. Korean J Radiol 2021:22:1569-1578.

4. Penin M, Martin MA, San Millan B, Garcia J. Learning curve of thyroid fine-needle aspiration biopsy. Endocrinol Diabetes Nutr 2017;64:539-543.

5. Baloch ZW, LiVolsi VA. Fine-needle aspiration of thyroid nodules: past, present, and future. Endocr Pract 2004;10:234-241.

6. Polyzos SA, Anastasilakis AD. Clinical complications following thyroid fine-needle biopsy: a systematic review. Clin Endocrinol (Oxf) 2009;71:157-165.

7. Turtulici G, Orlandi D, Corazza A, Sartoris R, Derchi LE, Silvestri E, et al. Percutaneous radiofrequency ablation of benign thyroid nodules assisted by a virtual needle tracking system. Ultrasound Med Biol 2014; $40: 1447-1452$. 
8. Freesmeyer M, Kuhnel C, Guhne F, Seifert P. Standard needle magnetization for ultrasound needle guidance: first clinical experiences in fine-needle aspiration cytology of thyroid nodules. J Ultrasound Med 2019;38:3311-3319.

9. Kopac DS, Chen J, Tang R, Sawka A, Vaghadia H. Comparison of a novel real-time SonixGPS needle-tracking ultrasound technique with traditional ultrasound for vascular access in a phantom gel model. J Vasc Surg 2013;58:735-741.

10. Auyong DB, Yuan SC, Rymer AN, Green CL, Hanson NA. A randomized crossover study comparing a novel needle guidance technology for simulated internal jugular vein cannulation. Anesthesiology 2015;123:535-541.

11. Kim EJ, Min J, Song J, Song K, Song JH, Byon HJ. The effect of electromagnetic guidance system on early learning curve of ultrasound for novices. Korean J Anesthesiol 2016;69:15-20.

12. Meiser VC, Kreysa H, Guntinas-Lichius O, Volk GF. Comparison of in-plane and out-of-plane needle insertion with vs. without needle guidance. Eur Arch Otorhinolaryngol 2016;273:2697-2705.

13. Nitsche JF, Shumard KM, Brost BC. Development and assessment of a novel task trainer and targeting tasks for ultrasound-guided invasive procedures. Acad Radiol 2017;24:700-708.

14. Watanabe K, Tokumine J, Lefor AK, Motoyasu A, Moriyama K, Yorozu T. A shallow angle short-axis out-of-plane approach reduces the rate of posterior wall injuries in central venous catheterization: a simulation study. Biomed Res Int 2018;2018:4793174.

15. Kasine T, Romundstad L, Rosseland LA, Ullensvang K, Fagerland MW, Hol PK, et al. Needle tip tracking for ultrasound-guided peripheral nerve block procedures: an observer blinded, randomised, controlled, crossover study on a phantom model. Acta Anaesthesiol Scand 2019;63:1055-1062.

16. Chew SC, Beh ZY, Hakumat Rai VR, Jamaluddin MF, Ng CC, Chinna $K$, et al. Ultrasound-guided central venous vascular access-novel needle navigation technology compared with conventional method: a randomized study. J Vasc Access 2020;21:26-32.

17. Kitahara CM, Sosa JA. The changing incidence of thyroid cancer. Nat Rev Endocrinol 2016;12:646-653.

18. Niazi AU, Chin KJ, Jin R, Chan VW. Real-time ultrasound-guided spinal anesthesia using the SonixGPS ultrasound guidance system: a feasibility study. Acta Anaesthesiol Scand 2014;58:875-881. 